\title{
Overexpression of OsLCT2, a Low-Affinity Cation Transporter Gene, Reduces Cadmium Accumulation in Shoots and Grains of Rice
}

\author{
Li Tang 1,2, Jiayu Dong ${ }^{2}$, Longtao Tan³ ${ }^{3}$ Zhongying Ji², Yaokui Li ${ }^{1}$, Yuantao Sun², Caiyan Chen³ ${ }^{3}$ Qiming LV ${ }^{1,2}$, \\ Bigang Mao ${ }^{1,2}$, Yuanyi Hu and Bingran Zhao ${ }^{1,2^{*}}$ (D)
}

\begin{abstract}
Cadmium (Cd)-contaminated rice is a serious issue affecting food safety. Understanding the molecular regulatory mechanisms of $\mathrm{Cd}$ accumulation in rice grains is crucial to minimizing $\mathrm{Cd}$ concentrations in grains. We identified a member of the low-affinity cation transporter family, OsLCT2 in rice. It was a membrane protein. OsLCT2 was expressed in all tissues of the elongation and maturation zones in roots, with the strongest expression in pericycle and stele cells adjacent to the xylem. When grown in Cd-contaminated paddy soils, rice plants overexpressing OsLCT2 significantly reduced $\mathrm{Cd}$ concentrations in the straw and grains. Hydroponic experiment demonstrated its overexpression decreased the rate of $\mathrm{Cd}$ translocation from roots to shoots, and reduced $\mathrm{Cd}$ concentrations in xylem sap and in shoots of rice. Moreover, its overexpression increased $\mathrm{Zn}$ concentrations in roots by up-regulating the expression of OsZIP9, a gene responsible for $Z n$ uptake. Overexpression of OsLCT2 reduces Cd accumulation in rice shoots and grains by limiting the amounts of $\mathrm{Cd}$ loaded into the xylem and restricting $\mathrm{Cd}$ translocation from roots to shoots of rice. Thus, OsLCT2 is a promising genetic resource to be engineered to reduce $C d$ accumulation in rice grains.
\end{abstract}

Keywords: Cadmium, OsLCT2, Rice, Root-to-shoot translocation, Overexpression, Metal transporter

\section{Introduction}

In recent years, the widespread contamination of farmland by toxic heavy metals has become an environmental problem, raising concerns about health risks to people all over the world. Cadmium (Cd) is one of the most toxic heavy metals to organisms. It has a long biological halflife (10-30 years) in the human body and is associated with various diseases, including renal damage, osteoporosis, and cancers (Godt et al. 2006; Uraguchi and Fujiwara 2013; Clemens and Ma 2016). Rice is the principal food source for over half of the global population and is prone to accumulate more $\mathrm{Cd}$ than other cereal crops (Zhao and Wang 2019). Chronic dietary consumption

\footnotetext{
*Correspondence: brzhaorice@163.com

1 State Key Laboratory of Hybrid Rice, Hunan Hybrid Rice Research Center,

Changsha 410125, China

Full list of author information is available at the end of the article
}

of rice with excessive $\mathrm{Cd}$ is the major source of human $\mathrm{Cd}$-intake for local residents of $\mathrm{Cd}$-contaminated areas (Hu et al. 2016). About $90 \%$ of rice is produced in Asia, and $\mathrm{Cd}$ contamination of rice has been reported in many Asian countries (Hu et al. 2016; Tang et al. 2017). Therefore, it is important and urgent to minimize Cd accumulation in rice grains for food safety and human health. Screening and breeding of rice cultivars with low $\mathrm{Cd}$ concentrations in the grains is regarded as the most costeffective and eco-friendly strategy (Chi et al. 2018).

Understanding the physiological and molecular mechanisms of $\mathrm{Cd}$ uptake, translocation, and distribution in rice is critical for developing rice cultivars with low $\mathrm{Cd}$ levels. Following $\mathrm{Cd}$ uptake from the soil by roots, $\mathrm{Cd}$ may be sequestered into the vacuoles of root cells or translocated from roots to shoots via xylem and then distributed/ redistributed to different organs via nodes (Clemens and Ma 2016). The uptake and transport of $C d$ is mediated by 
transporters of divalent metals, such as manganese $(\mathrm{Mn})$, iron $(\mathrm{Fe})$, zinc $(\mathrm{Zn})$, and calcium $(\mathrm{Ca})$, which show chemical characteristics similar to those of $\mathrm{Cd}$. To date, several transporter families have been reported involved in this process in rice, including the natural resistance-associated macrophage protein (NRAMP) family, the zincregulated/iron-regulated transporter-like protein (ZIP) family, and heavy metal ATPase (HMA) family.

OsNRAMP5 is the major transporter responsible for the uptake of both $\mathrm{Cd}$ and $\mathrm{Mn}$, and the knockout of $O s N$ RAMP5 dramatically reduces $\mathrm{Cd}$ and $\mathrm{Mn}$ concentrations in rice (Ishikawa et al. 2012; Sasaki et al. 2012). Mutation of OsNRAMP5 via the CRISPR/Cas9 system produced a low $\mathrm{Cd}$-accumulating indica hybrid rice without compromising its grain yield normally (Tang et al. 2017). However, osnramp 5 mutants exhibited impaired growth under conditions of low Mn supply caused by Mn deficiency in plants (Yang et al. 2014). In addition, iron-regulated transporters OsIRT1 and OsIRT2 and Mn transporters OsNRAMP1 and OsCd1 (a member of a major facilitator family) also participate in Cd uptake in rice, but their contributions are relatively minor compared to that of OsNRAMP5 (Nakanishi et al. 2006; Senoura et al. 2011; Yan et al. 2019; Chang et al. 2020).

Once $\mathrm{Cd}$ enters into the roots, it moves radially into the stele for xylem loading and then transfers to shoots mainly via the xylem. In the process of radial transport of $\mathrm{Cd}$ in roots, chelation, compartmentalization, and adsorption retain most of the $\mathrm{Cd}$ in roots and consequently restrict $\mathrm{Cd}$ translocation from roots to shoots (Nocito et al. 2011). OsHMA3 is localized to the tonoplast of root cells and compartmentalizes Cd into vacuoles, thus limiting the quantities of $\mathrm{Cd}$ loaded into the xylem (Ueno et al. 2010; Miyadate et al. 2011; Sasaki et al. 2014). Previous studies have identified two lossof-function alleles of OsHMA3 harboring a substitution at amino acid position 80 or 380 and one weak allele of OsHMA3 carrying an amino acid mutation at position 512. Rice varieties with the above alleles of OsHMA3 had high $\mathrm{Cd}$ concentrations in the shoots and grains of rice due to a weakened ability to sequester $\mathrm{Cd}$ in the root vacuoles (Ueno et al. 2010; Yan et al. 2016; Sun et al. 2019).

Several transporters, including OsHMA2, OsZIP7, and cation/Ca exchanger 2 (OsCCX2), have been reported to facilitate the root-to-shoot translocation of $\mathrm{Cd}$ and delivery of $\mathrm{Cd}$ to developing tissues and grains (Takahashi et al. 2012; Yamaji et al. 2013; Hao et al. 2018; Tan et al. 2019). All of them are not Cd-specific transporters. Among them, OsHMA2 and OsZIP7 showed transport activity of $\mathrm{Zn}$ and $\mathrm{Cd}$, and OsCCX2 showed efflux transport activity of $\mathrm{Ca}$ and $\mathrm{Cd}$. Knockout of OsHMA2 or OsZIP7 resulted in severe reductions of grain yield and was mainly attributed to $\mathrm{Zn}$ deficiency in the plants
(Yamaji et al. 2013; Tan et al. 2019). Knockout of OsCCX2 reduced the 1000-grain weight, which was attributed to decreased Ca transport (Hao et al. 2018). A defensin-like protein in rice, OsCAL1, can chelate $\mathrm{Cd}$ and assist $\mathrm{Cd}$ secretion to apoplastic spaces, and consequently accelerates long-distance $\mathrm{Cd}$ transport via the xylem (Luo et al. 2018). Knockout of OsCAL1 reduced Cd translocation to shoots, but it did not affect $\mathrm{Cd}$ concentrations in the grains of rice (Luo et al. 2018).

$\mathrm{Cd}$ can be delivered to grains via phloem transport, which is regulated by OsHMA2 and low-affinity cation transporter 1 (OsLCT1). OsHMA2 is implicated in reloading $\mathrm{Zn}$ and $\mathrm{Cd}$ from the parenchyma tissues into the phloem of diffuse vascular bundles (Yamaji et al. 2013). OsLCT1 is the only member of the LCT family in rice that has been identified and functionally characterized. OsLCT1 is a plasma membrane-localized efflux transporter of $\mathrm{Cd}$, potassium $(\mathrm{K}), \mathrm{Ca}$, magnesium $(\mathrm{Mg})$ and $\mathrm{Mn}$. In the uppermost node, OsLCT1 was expressed around the enlarged vascular bundles and diffused vascular bundles. Knock down of OsLCT1 by RNAi did not affect the $\mathrm{Cd}$ concentration in xylem sap, but it did decrease the $\mathrm{Cd}$ concentration in phloem sap, strongly suggesting that OsLCT1 is involved in xylem-to-phloem transfer of $\mathrm{Cd}$. These RNAi lines also showed about a $50 \%$ reduction of $\mathrm{Cd}$ in grains without affecting mineral nutrient concentrations and plant growth (Uraguchi et al. 2011).

Compartmentalization of metal in the vacuole, endoplasmic reticulum (ER), or Golgi apparatus by endomembrane-localized transporters is crucial for plant tolerance to the stress of excess metal (Shao et al. 2017; De Caroli et al. 2020). However, OsHMA3 is the only identified intracellular transporter that sequesters $\mathrm{Cd}$ in the vacuoles of roots in rice. The functions and molecular mechanisms of endomembrane-localized Cd transporters in rice are poorly understood. In this study, we cloned OsLCT2, a novel member of the $L C T$ family in rice, and found that its GFP-fusion protein was localized to the ER when transiently expressed by the $35 \mathrm{~S}$ promoter. Overexpression of OsLCT2 weakened Cd translocation from roots to shoots and consequently reduced $\mathrm{Cd}$ concentrations in rice shoots and grains.

\section{Results}

Cloning and Characterization of OsLCT2

Rice OsLCT1 is an important Cd transporter (Uraguchi et al. 2011, 2014). Unexpectedly, no OsLCT1 fragments could be obtained by PCR amplification from the DNA of indica rice cultivars, Huazhan, 93-11 and Shuhui 498. Hence, BLAST searches were conducted using the amino acid sequences of OsLCT1 against the genome of the rice cultivar Shuhui498 in the National Center 
for Biotechnology Information (NCBI) database. The OsLCT1 sequence could not be aligned, confirming the absence of OSLCT1 in the genome of Shuhui498. However, we found a putative rice LCT protein, designated as OsLCT2. Because the full-length cDNA sequence of OsLCT2 was not available in the Rice Annotation Project Database (http://rapdb.dna.affrc.go.jp/), we performed $5^{\prime}$ - and $3^{\prime}$ - rapid amplification of cDNA ends (RACE) to clone the full-length cDNA of OsLCT2 (GenBank accession number: MW757982) from the indica rice cultivar Huazhan.

OsLCT2 contains three exons and two introns (Fig. 1a), and encodes a peptide of 478 amino acids. It is located at position $23,774,023$ to $23,776,721$ on chromosome 6 in the Shuhui498 genome. Similar to TaLCT1 and OsLCT1, OsLCT2 has a hydrophilic amino $\mathrm{N}$ terminus containing sequences enriched in Pro, Ser, Thr, and Glu, collectively known as the PEST sequence (Fig. 1b) (Kennedy and Rinne 1997). Both the TMPRED and CCTOP programs predicted that OsLCT2 is a membrane protein with eleven transmembrane domains (Fig. 1b). Phylogenetic analysis showed that LCT-like proteins were only found in grass plants (Fig. 1c). Among those LCT members in grass plants, the closest homolog of OsLCT2 was TaLCT1, which also belonged to the same subgroup as OsLCT2 (Fig. 1c). Amino acid sequence alignment revealed that OsLCT2 shared 55\% and 33\% sequence identity with TaLCT1 and OsLCT1, respectively. To explore the evolutionary relationship between OsLCT2 and OsLCT1, phylogenetic analysis was conducted using their predicted amino acid sequences and LCT-like proteins in eight wild Oryza species. OsLCT2 and its orthologs in Oryza rufipogon were in the same clade with 98.5\% sequence identity, whereas the OsLCT1 showed high similarity to the LCT-like proteins in Oryza barthii, Oryza glaberrima, and Oryza nivara, with 98.6\%, 98.6\%, and $95.7 \%$ sequence identity, respectively (Additional file 1: Fig. S1a). These results revealed that orthologs of both OsLCT2 and OsLCT1 existed in wild rice species, and the considerable sequence divergence between
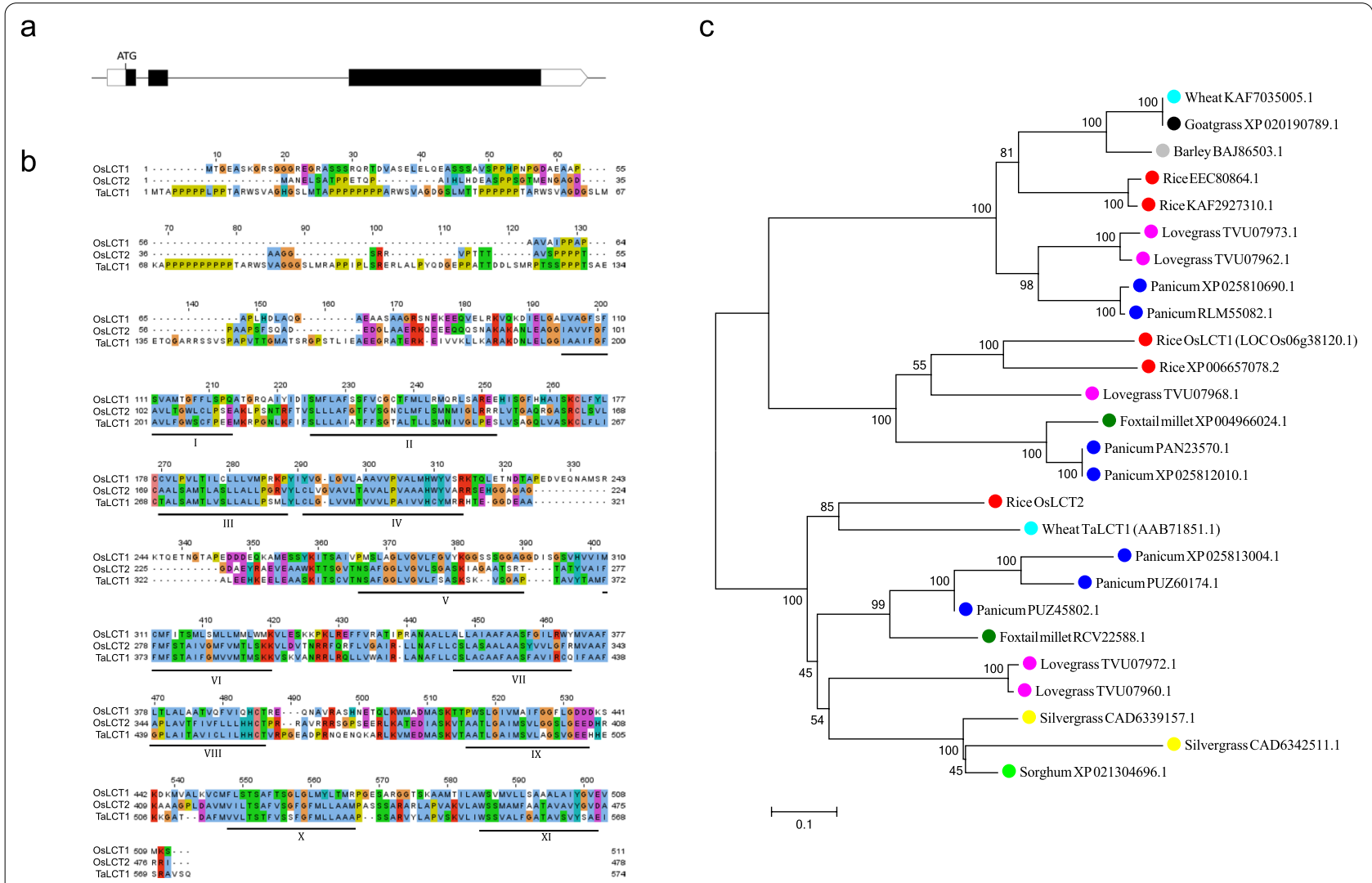

Fig. 1 Gene structure of OsLCT2 and phylogenic relationships of LCT proteins. a Schematic diagram of OsLCT2 gene structure. UTRs, exons, and introns are indicated by blank rectangles, black rectangles, and black lines, respectively. b Alignment of the predicted amino acid sequences of OsLCT1, OsLCT2 and TaLCT1. Identical or similar amino acids are indicated by the same background color. Non-conservative amino acids are indicated by different background colors. The predicted trans-membrane domains are underlined and labeled by Roman numerals. c Phylogenic tree of LCT-like proteins. The predicted LCT amino acid sequences were used to construct the phylogenetic tree using MEGA7. Unidentified proteins are represented by accession numbers in the NCBI database. The scale bar of 0.1 is equal to $10 \%$ sequence divergence 
OsLCT2 and OsLCT1 derived from the divergence of their ancestor gene pools. This finding implied that they diverged before the diversification of Oryza genus.

We further analyzed natural variations of OsLCT2 coding sequence (CDS) using 3,620 rice accessions of the MBKbase-rice database (Peng et al. 2020). Twelve single-nucleotide polymorphisms (SNPs) were detected in the OsLCT2 CDS region (Additional file 1: Fig. S1b). Based on these variations, its CDS region was classified into nine haplotypes (rare haplotypes of $<10$ accessions were not shown). Haplotype 1 , which was harbored in Huazhan and Shuhui 498, was also prevalent in the four main rice subpopulations, accounting for $68.3 \%$ of indica, $43.3 \%$ of temperate japonica, $72.3 \%$ of tropical japonica, and $39.6 \%$ of aus (Additional file 1: Fig. S1b, c). Notably, there were different proportions of OsLCT2 deletions in these rice subpopulations. OsLCT2 was absent in about $54 \%$ of temperate japonica and aus, but only in a small fraction of indica (4.3\%) and tropical japonica (14.3\%) (Additional file 1: Fig. S1b, c).

\section{Expression Pattern of OsLCT2}

The expression profiles of OsLCT2 in rice seedlings were investigated by quantitative real-time PCR (qRT-PCR). The expression level of OsLCT2 in roots was relatively high, followed by leaves, with the lowest expression level in the basal region ( $2 \mathrm{~cm}$ above the roots) (Fig. 2a). To investigate the effects of metal deficiency and excess on the expression of $O s L C T 2$, rice plants were grown in nutrient solutions either free of $\mathrm{Fe}, \mathrm{Mn}$, or $\mathrm{Zn}$ or containing high concentrations of $\mathrm{Fe}, \mathrm{Mn}$, or $\mathrm{Zn}$ for $7 \mathrm{~d}$. Expression of OsLCT2 was unaffected by the deficiency of those metals, while it was induced by excess Fe and Zn (Fig. 2b). To determine the responses of OsLCT2 to Cd stress, rice seedlings were treated with $0,0.5,2.5$ or $25 \mu \mathrm{M} \mathrm{Cd}$ for $24 \mathrm{~h}$. The OsLCT2 expression in roots was induced by $\mathrm{Cd}$ in a dose-dependent manner. Its transcripts increased by about fourfold in response to $25 \mu \mathrm{M} \mathrm{Cd}$, but slightly increased in response to $2.5 \mu \mathrm{M} \mathrm{Cd}$ concentrations when compared with that in the absence of $\mathrm{Cd}$ (Fig. 2c).

To analyze the tissue specificity of OsLCT2 expression, we determined OsLCT2 promoter activity by assessing $\beta$-glucuronidase (GUS) activity histochemically in transgenic rice plants expressing the GUS gene driven by the OSLCT2 promoter. The GUS staining was mainly observed in the elongation and maturation zones of the root but not in the meristematic zone and root cap (Fig. 2d I, II). In the cross sections and vertical sections of the elongation and maturation zone of the primary root, GUS activity appeared in all the tissues, including epidermis, cortex and stele, with the strongest signal in pericycle and stele cells adjacent to the xylem (Fig. 2d IV-VII). As the primary root matured, the GUS staining faded away, except for a pronounced staining in the lateral roots (Fig. 2d I, III). The observations in the cross sections and vertical sections of the maturation zone where the lateral roots began to form showed that GUS signal occurred mainly in the stele of the primary and lateral roots, especially the undifferentiated vascular tissue of the lateral roots (Fig. 2d VI-VII).

\section{Subcellular Localization of OsLCT2}

To detect the subcellular localization of the OsLCT2 protein, OsLCT2 was separately fused to the $\mathrm{N}$-terminus or C-terminus of an enhanced GFP (eGFP) via a 16 aminoacid linker. OsLCT2-eGFP or $e G F P-O s L C T 2$ driven by the constitutive cauliflower mosaic virus $35 \mathrm{~S}$ promoter were cloned into a transient expression vector and introduced into rice leaf protoplasts. Both OsLCT2-eGFP and eGFPOsLCT2 fusion proteins were observed at the ER, as evidenced by their fluorescence signals overlapping with the ER retention signal His-Asp-Glu-Leu (HDEL) fused with the red fluorescent protein mCherry (Fig. 3). In contrast, the control vector (containing eGFP alone driven by the $35 \mathrm{~S}$ promoter) produced green fluorescence in the cytosol and nucleus (Fig. 3).

\section{Overexpression of OsLCT2 Reduces Cd Concentration in Rice Grains}

To investigate the physiological role of OsLCT2 in rice, we generated two independent overexpression lines driven by a maize ubiquitin promoter and two independent knockout lines using CRISPR/Cas9 technology. The two overexpression lines greatly enhanced expression of OsLCT2, compared with that of wild-type (WT) plants, according to the qRT-PCR analysis (Fig. 4a). Sequencing confirmed that each knockout line had insertions that caused a frameshift of the coding sequence (Fig. 4b).

When plants were grown in a field contaminated with $0.65 \mathrm{mg} / \mathrm{kg} \mathrm{Cd}$, overexpressing OsLCT2 tended to reduce grain yield compared with that of the WT, although the difference was only statistically significant in one overexpression line (Fig. 4c, e; Additional file 1: Fig. S2). In contrast, no apparent changes of morphological phenotype or grain yield were found between the knockout lines and the WT (Fig. 4d, e). We then measured Cd, Mn, Fe, $\mathrm{Zn}$ and $\mathrm{Cu}$ accumulation in these plants at the maturity stage to evaluate whether OsLCT2 affected metal accumulation in plants grown in field conditions. The $\mathrm{Cd}$ concentrations in the brown rice and straw of the overexpression lines were significantly lower by $24.1-27.5 \%$ and $30.7-37.5 \%$, respectively, compared with those of the WT (Fig. 4f, k). These results suggest that OsLCT2 overexpression may not only affect $\mathrm{Cd}$ in the grain but also in all above-ground tissues. However, the $\mathrm{Mn}, \mathrm{Fe}, \mathrm{Zn}$ and $\mathrm{Cu}$ concentrations in brown rice and straw were similar 
a

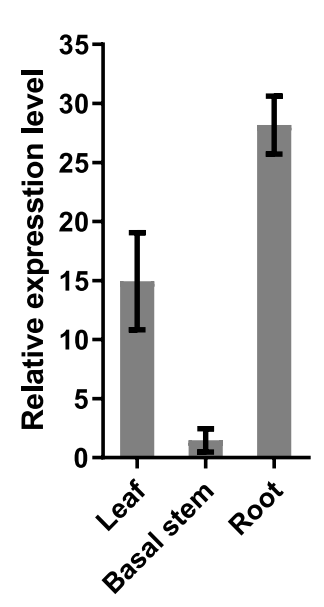

b

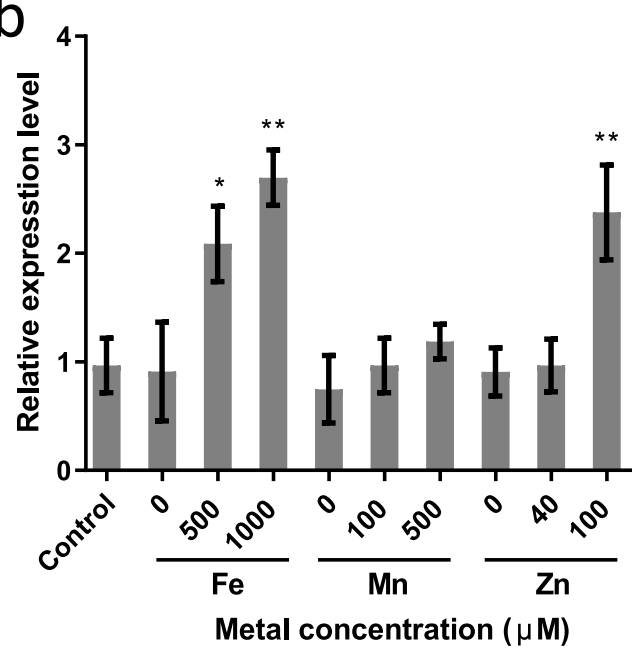

$\mathrm{C}$

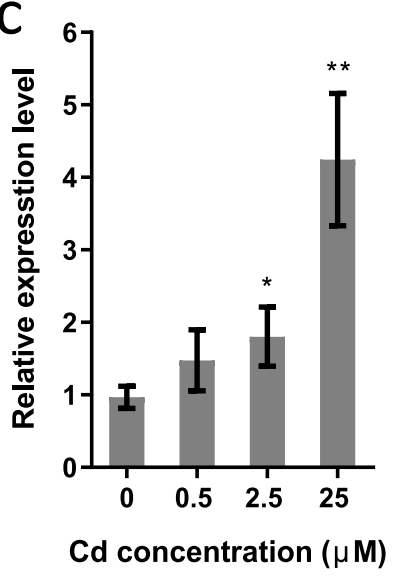

d
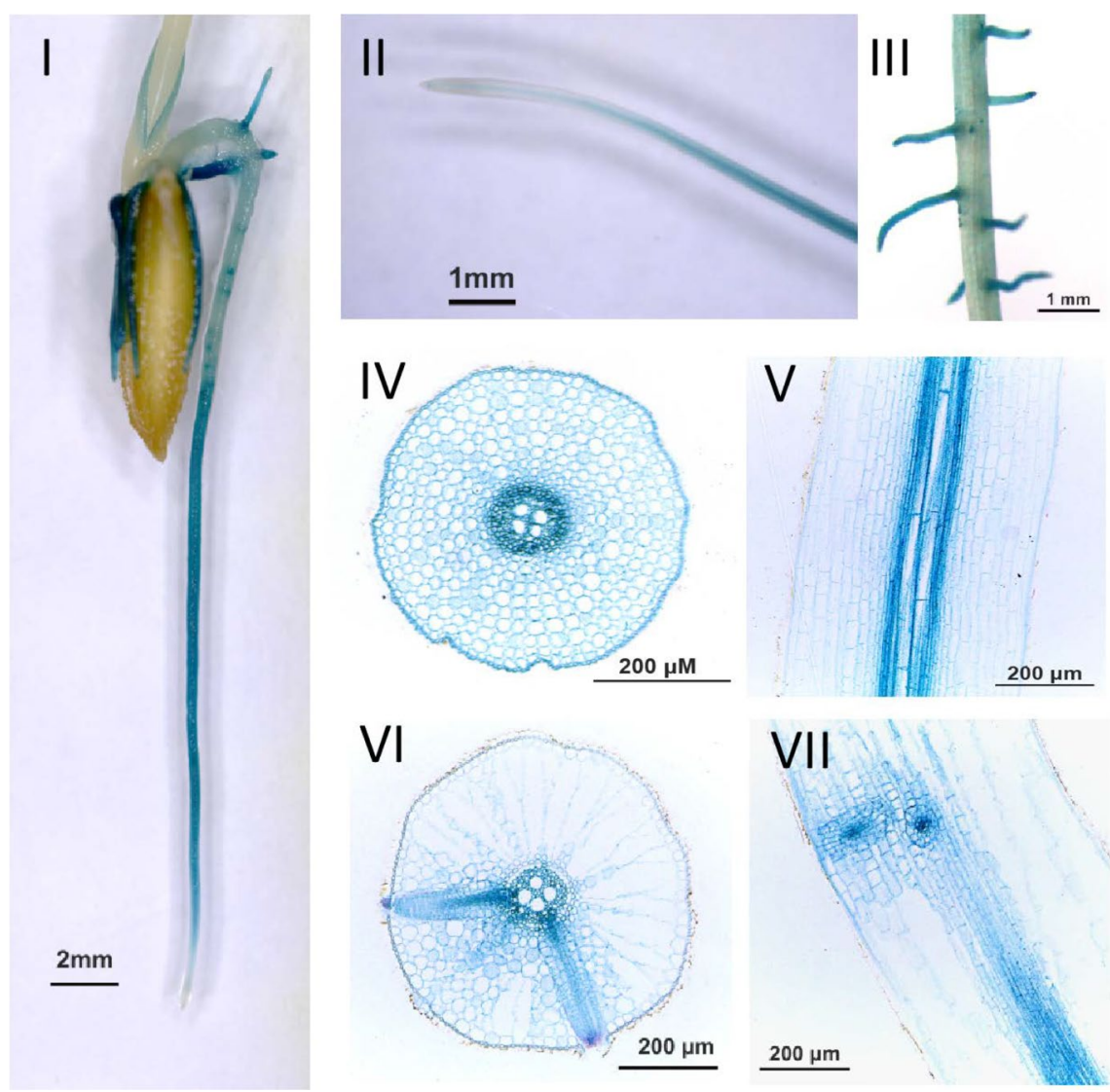

VI
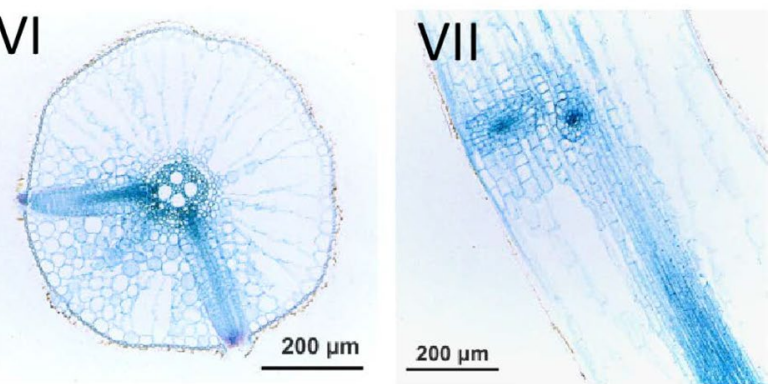

Fig. 2 Expression patterns of OsLCT2. a-c The expression of OsLCT2 was determined by qRT-PCR. a Tissue-dependent expression of OsLCT2 in 2-week-old seedlings. The leaf, root and basal stem ( $2 \mathrm{~cm}$ above the roots) were sampled for expression analysis. Relative expression of OSLCT2 is shown, with its expression in the basal stem set to 1. $\mathbf{b}$ Response of OsLCT2 in seedling roots to the absence or excess of Fe, Mn or Zn. Seven-day-old seedlings were grown in Yoshida nutrient solution without Fe, Mn, or Zn or with high concentrations of Fe, Mn, or Zn for $7 \mathrm{~d}$. Seedlings grown in standard Yoshida nutrient solution were used as controls. c Response of OsLCT2 in seedling roots to external Cd. Two-week-old seedlings were exposed to different concentrations of $\mathrm{Cd}$ for $24 \mathrm{~h}$. Data show means \pm SD of three biological replicates. In $\mathbf{b}$ and $\mathbf{c}$, asterisks above the bars indicate significant differences between the treatments and controls $\left({ }^{*} P<0.05\right.$, ${ }^{*} P<0.01$; Student's $t$-test). $\mathbf{d}$ Histochemical GUS staining of roots of transgenic rice plants transformed with OSLCT2 promoter-driven GUS constructs. Tissue-specific expression of OSLCT2 in the entire roots (I), root tip (II), lateral roots (III), root elongation zone (IV, V), and maturation zone (VI, VII) visualized in cross section (IV, VI) and vertical section (V, VII) 

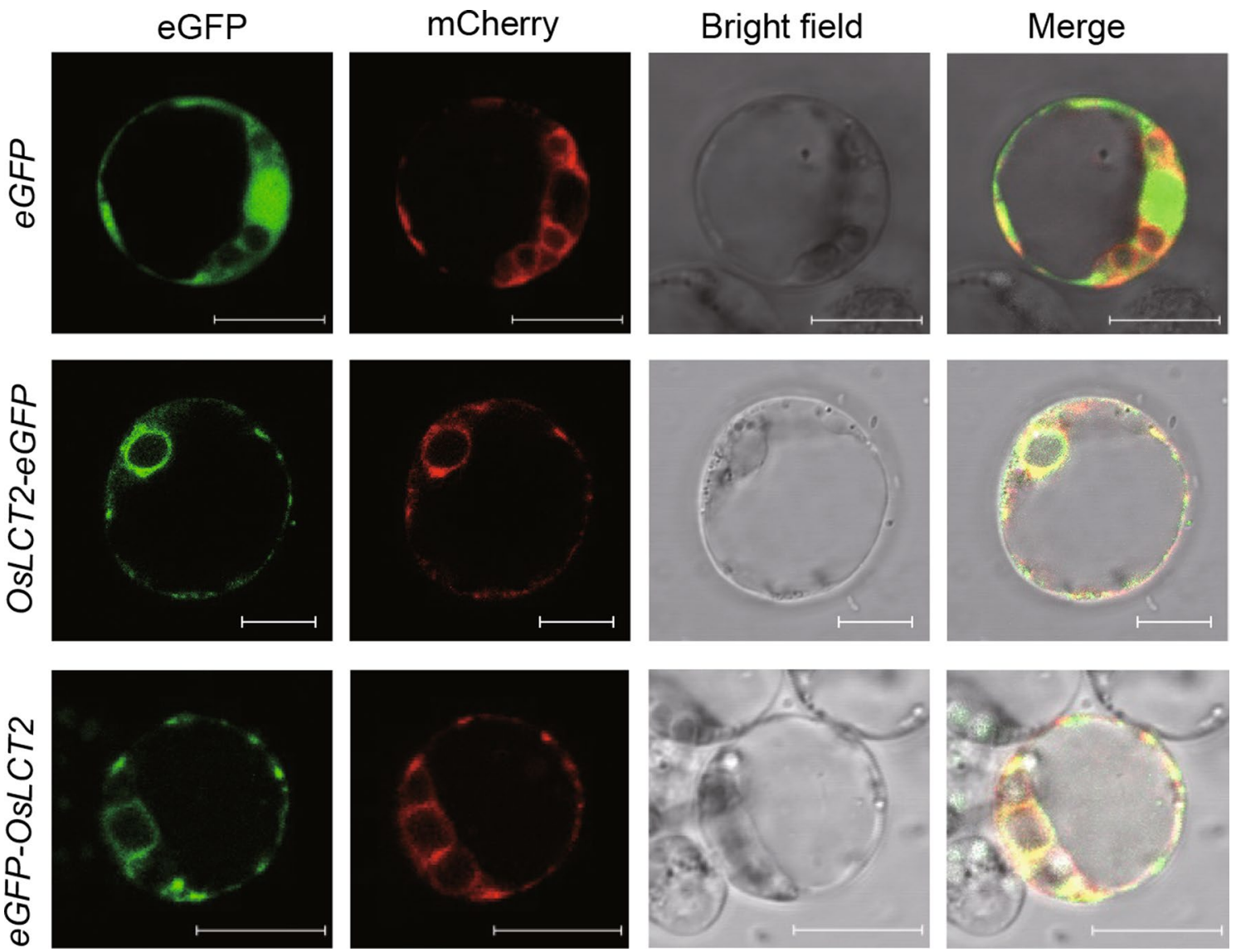

Fig. 3 Subcellular localization of OSLCT2. eGFP, OsLCT2-eGFP or OsLCT2-eGFP was co-expressed with HDEL-mCherry, an endoplasmic reticulum localization marker, in rice protoplasts. The upper panels show the localization of eGFP and HDEL-mCherry as controls. The middle panels show the localization of OsLCT2-eGFP and HDEL-mCherry. The lower panels show the localization of eGFP- OsLCT2 and HDEL-mCherry. Scale bar, $10 \mu m$

between the overexpression lines and WT (Fig. 4g-j, $1-o)$. In comparison, all of the above metal concentrations in brown rice and straw did not differ significantly between the knockout lines and the WT (Fig. 4f-o).

\section{Overexpression of OsLCT2 Weakens Root-to-Shoot Translocation of $\mathrm{Cd}, \mathrm{Mn}$, Fe and $\mathrm{Zn}$}

To understand the cause of OSLCT2 overexpression reducing $\mathrm{Cd}$ concentrations in rice straw and grains, twoweek-old seedlings of hydroponically-grown OsLCT2 overexpression lines and WT plants were transferred into nutrient solutions containing $0,0.5$ or $1 \mu \mathrm{M} \mathrm{Cd}$ and kept in solutions for 14 days. Growth responses and divalent metal concentrations in shoots and roots of the overexpression lines and WT were investigated. OsLCT2 overexpression lines and WT plants showed comparable growth within each of the 0 and $0.5 \mu \mathrm{M} \mathrm{Cd}$ treatments, while shoots of overexpression lines were $7-8 \%$ longer and $13-17 \%$ heavier (dry weight) in the $1 \mu \mathrm{M} \mathrm{Cd}$ treatment (Fig. 5a-d). The results suggest that overexpressing
OsLCT2 does not affect seedling growth and enhances rice tolerance to excess $\mathrm{Cd}$ under hydroponic culture with sufficient mineral nutrition.

Analysis of metal concentrations showed that there were no differences in $\mathrm{Mg}, \mathrm{Ca}$ and $\mathrm{Cu}$ concentrations in both the shoots and roots between the overexpression lines and WT plants (Fig. 5e-h), while the Mn, Fe, $\mathrm{Zn}$ concentrations in shoots were significantly lower in the overexpression lines than in the WT with and without Cd stress (Fig. 5f; Additional file 1: Fig. S3a-c). The $\mathrm{Mn}$ and Fe concentrations in roots were similar between the WT plants and overexpressing lines, whereas the $\mathrm{Zn}$ concentration in roots was significantly higher in the overexpression lines than in the WT plants with or without Cd treatment (Fig. 5h; Additional file 1: Fig. S3d, e). Under hydroponic conditions without $\mathrm{Cd}$, growth of the overexpression lines was similar to that of the WT plants (Fig. 5a), suggesting that the reduced concentrations of $\mathrm{Mn}, \mathrm{Fe}$, and $\mathrm{Zn}$ in shoots were sufficient for the normal growth and development of overexpression lines. 


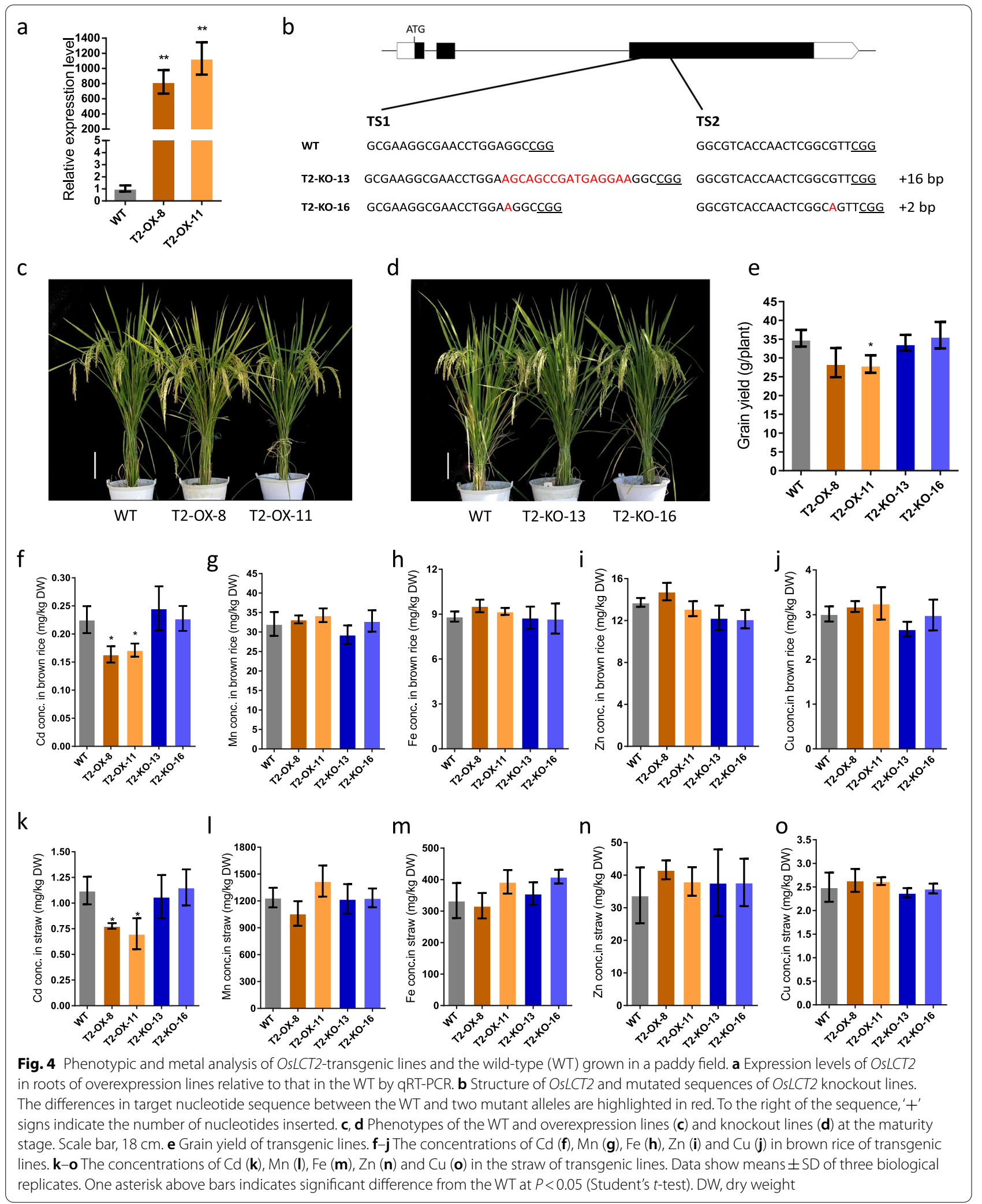


In the presence of 0.5 and $1 \mu \mathrm{M} \mathrm{Cd}$, OsLCT2 overexpression lines exhibited $21.8-26.9 \%$ and $26.7-28.1 \%$ lower $\mathrm{Cd}$ concentrations in shoots, respectively, but similar $\mathrm{Cd}$ concentrations in roots compared to those of the WT (Fig. 5i, j). In the presence of $1 \mu \mathrm{M} \mathrm{Cd}$, overexpression lines showed better Cd tolerance than the WT, which might be attributed to the lower Cd concentrations in shoots that reduced the inhibitory effect of $\mathrm{Cd}$ on growth of plants (Fig. 5a). Besides, the overexpression lines showed lower $\mathrm{Cd}, \mathrm{Mn}, \mathrm{Fe}$ and $\mathrm{Zn}$ concentrations in the xylem sap than that of the WT (Fig. 5k). As a result, its overexpression significantly decreased the percentages of $\mathrm{Cd}, \mathrm{Mn}, \mathrm{Fe}$ and $\mathrm{Zn}$ translocated from roots to shoots (Fig. 5l). Among them, the proportion of Mn transported to the shoots was slightly lower, while the proportions of $\mathrm{Cd}, \mathrm{Zn}$ and Fe transported to the shoots were moderately lower than those of the WT (Fig. 5l). These results indicate that OsLCT2 has poor ability to discriminate among the divalent metal cations $\mathrm{Cd}, \mathrm{Mn}, \mathrm{Fe}$ and $\mathrm{Zn}$. Its overexpression weakens the translocation of these four metals from roots to shoots by limiting their amounts loaded into the xylem.

\section{Effect of OsLCT2 Overexpression on Expression of OsZIP Genes}

Because the ZIP family displays transport activity of various cations, including $\mathrm{Zn}, \mathrm{Fe}, \mathrm{Mn}$ and $\mathrm{Cd}$ (Lee and $\mathrm{An}$ 2009; Ajeesh Krishna et al. 2020), we examined expression of ten genes belonging to the OsZIP family in roots of OsLCT2 overexpression lines and the WT in the presence or absence of $0.5 \mu \mathrm{M} \mathrm{Cd}$ for $24 \mathrm{~h}$. Among the genes, the expression levels of OsIRT1, OsIRT2, OsZIP5, OsZIP6 and OsZIP9 were upregulated by $\mathrm{Cd}$ in both the WT and OsLCT2 overexpression lines (Fig. 6). The expression levels of most OsZIP genes did not differ between the OsLCT2 overexpression and WT plants, with the exception of OsZIP9 expression, which was significantly higher in the overexpression lines than in the WT, irrespective of Cd stress (Fig. 6). OsZIP9 was recently reported to play an important role in the uptake of $\mathrm{Zn}$ in rice (Huang et al. 2020; Tan et al. 2020; Yang et al. 2020), so the increased concentrations of $\mathrm{Zn}$ in roots of overexpression lines may have been caused by the up-regulation of OsZIP9 expression.

\section{Discussion}

\section{OsLCT2 is a Novel LCT Transporter in Rice}

To date, rice OsLCT1 and wheat TaLCT1 are the only two LCT members that have been reported in the LCT family. In this study, we characterized OsLCT2 as a new member of the LCT family. Like OsLCT1 and TaLCT1, OsLCT2 is a typical transmembrane protein harboring PEST sequences at the $\mathrm{N}$ terminus (Fig. 1b). Previous reports demonstrated that TaLCT1 exhibited influx activity of Cd, Ca, K and $\mathrm{Na}$ (Kennedy and Rinne 1997; Clemens et al. 1998), whereas OsLCT1 exhibited efflux activity of $\mathrm{Cd}, \mathrm{Mg}, \mathrm{Ca}, \mathrm{Mn}$ and $\mathrm{K}$ (Uraguchi et al. 2011, 2014). Likewise, OsLCT2 may have broad substrate specificity in transporting Cd, Mn, Fe and Zn (Fig. 5).

We determined that OsLCT2 and OsLCT1 have 33\% sequence identity and belong to different subgroups of the LCT family (Fig. 1b, c). In the Nipponbare genome, OsLCT2 is absent, whereas OsLCT1 is located at position $22,566,775-22,571,982$ on chromosome 6 . We aligned the region ranging from $20.0 \mathrm{Mb}$ to $24.0 \mathrm{Mb}$ on chromosome 6 of Nipponbare to Shuhui498 genome. As a result, we found that the $0.5 \mathrm{M}$ interval (from 22.5 to $23.0 \mathrm{Mb}$ ) encompassing OsLCT1 on chromosome 6 of Nipponbare had extensive sequence divergence from the corresponding interval containing OSLCT2 on chromosome 6 of Shuhui498 (Additional file 1: Fig. S4). The results of the genome alignment suggested that OsLCT1 and OsLCT2 were located in the same genomic region of chromosome 6 , but there were large fragments variation in this region, resulting in the low sequence similarity observed between OsLCT1 and OsLCT2. Although OsLCT2 had 9 haplotypes, haplotype 1 in Huazhan and Shuhui498 was the only major allele, whereas other alleles were rare (Additional file 1: Fig. S1b, c). Moreover, OsLCT2 was absent from the genomes of some rice varieties. Compared with indica and tropical japonica, temperate japonica and aus rice exhibited much higher incidence of OsLCT2 deletion. (Additional file 1: Fig. S1b, c). In these rice varieties lacking OsLCT2, OsLCT1 might be present in their genomes.

TaLCT1 is the closest homolog of OsLCT2, and they both belong to the same subgroup with $55 \%$ sequence identity (Fig. 1b, c). OsLCT1 is localized to the plasma membrane and its transcripts are mainly found in nodes and leaf blades during the grain-ripening stage, playing an

\section{(See figure on next page.)}

Fig. 5 Phenotypic and metal analysis of OSLCT2 overexpression lines and the WT grown hydroponically. Two-week-old seedlings grown hydroponically were exposed to 0, 0.5 or $1 \mu \mathrm{M}$ Cd for two weeks. a Phenotypes of the WT and overexpression lines. Scale bar, $3 \mathrm{~cm}$. b-d Shoot length (b), shoot dry weight (c) and root dry weight (d) of overexpression lines and the WT. e-h Metal concentrations in shoots (e, $\mathbf{f})$ and roots ( $\mathbf{g}$, h) of overexpression lines and the WT without $\mathbf{C d}$ treatment. $\mathbf{i}$, $\mathbf{j}$ Concentrations of $\mathbf{C d}$ in shoots (i) and roots (j) of overexpression lines and the WT at 0.5 or $1 \mu \mathrm{M}$ Cd supply. $\mathbf{k}$. I Metal concentrations in xylem sap (k) and metal translocation rates from roots to shoot (I) of overexpression lines and the WT at $0.5 \mu \mathrm{M}$ Cd supply. Data show means \pm SD of three biological replicates. Asterisks above the bars indicate significant differences from the WT $\left({ }^{*} P<0.05,{ }^{* *} P<0.01\right.$; Student's $t$-test). DW, dry weight 
a
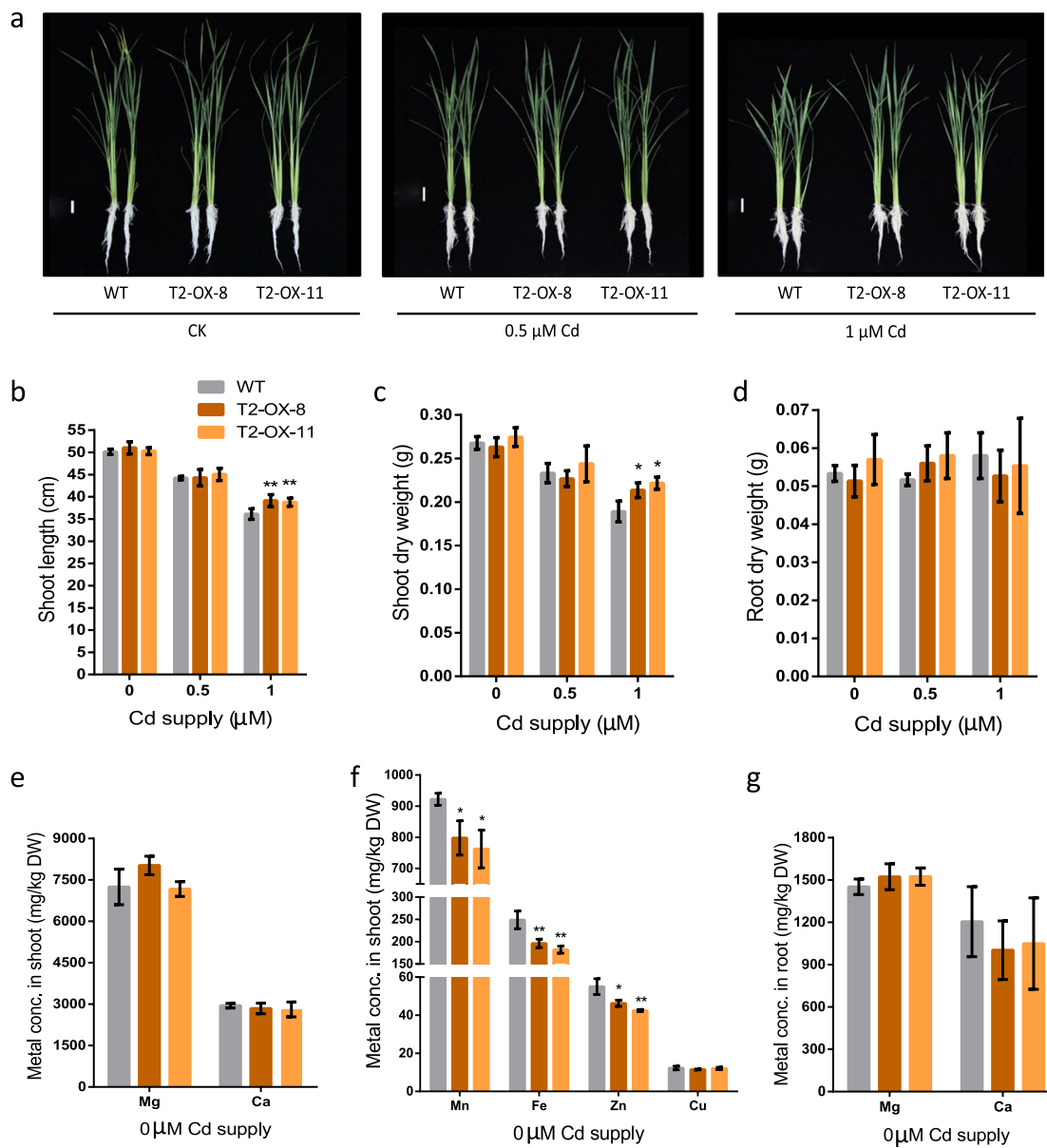

g
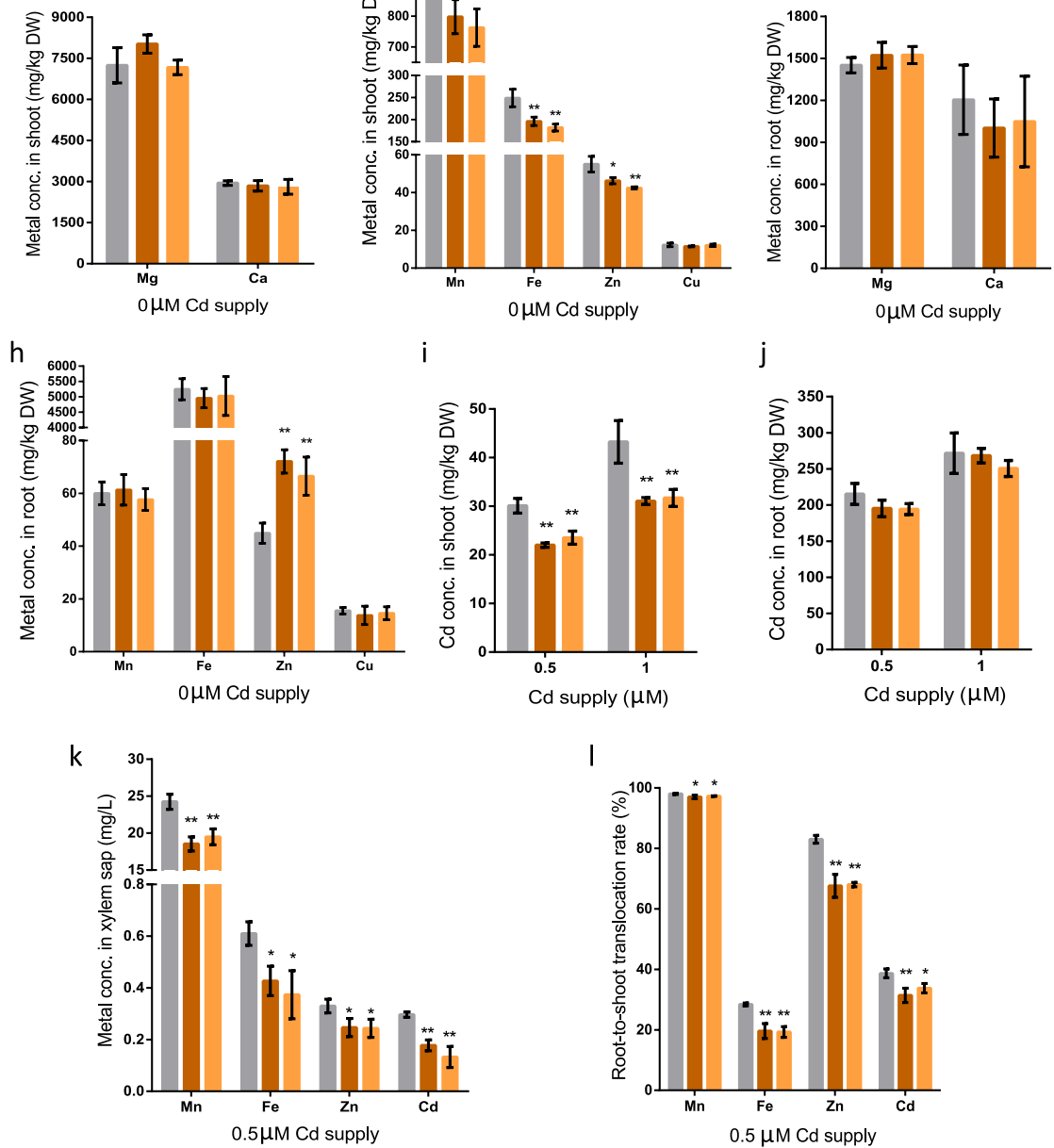

Fig. 5 (See legend on previous page.) 

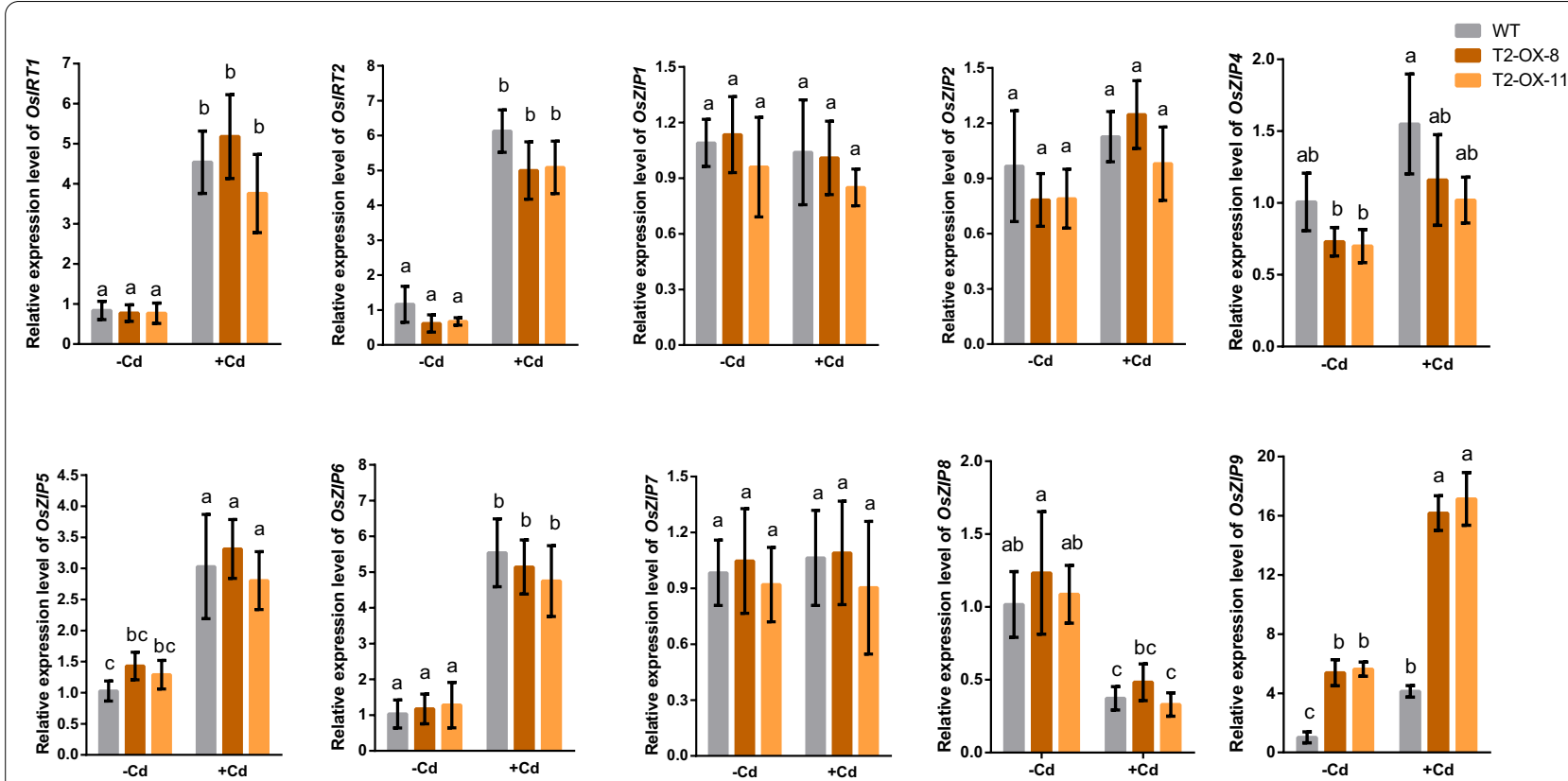

Fig. 6 Expression of ten OsZIP genes in roots of OsLCT2 overexpression lines and the WT. Seedlings were exposed to 0 or $0.5 \mu \mathrm{M} C \mathrm{~d}$ for $24 \mathrm{~h}$. Expression levels were determined by qRT-PCR. Expression levels relative to WT without $\mathrm{Cd}$ treatment are shown as means \pm SD of three biological replicates. Different letters above the bars indicate significant differences at $P<0.05$ (one-way ANOVA, Tukey's test)

important role in regulation of $\mathrm{Cd}$ loading to the phloem and $\mathrm{Cd}$ concentrations in grains of rice (Uraguchi et al. 2011). TaLCT1 is expressed in wheat roots and leaves (Kennedy and Rinne 1997). However, no study has investigated the phenotypes of TaLCT1 transgenic lines, so its function in planta is unclear. OsLCT2 is also expressed in roots and leaves (Fig. 2a), and this protein might be localized to the ER (Fig. 3). So OsLCT2 has completely distinct features in amino acid sequences, expression pattern, and subcellular localization from those of OsLCT1.

\section{Overexpression of OsLCT2 Reduces xylem-Mediated Cd Translocation from Roots to Shoots}

Root-to-shoot translocation via the xylem is considered as a critical process determining the accumulation of $\mathrm{Cd}$ in shoots and grains of rice (Uraguchi et al. 2009). Therefore, it is essential to study the factors controlling $\mathrm{Cd}$ translocation to shoots in order to minimize $\mathrm{Cd}$ accumulation in grains. The total amount of Cd transported by this process depends mainly on two aspects, one is the efficiency of xylem loading and velocity of xylem transport, and the other is the ability of roots to retain $\mathrm{Cd}$. In roots, $\mathrm{Cd}$ appears to use the $\mathrm{Zn}$ - and Ca-transport systems to translocate to shoots. The Zn transporters OsHMA2 and OsZIP7 and Ca transporter OsCCX2 have been shown to mediate root-toshoot translocation of Cd (Satoh-Nagasawa et al. 2012;
Takahashi et al. 2012; Yamaji et al. 2013; Hao et al. 2018; Tan et al. 2019). OsHMA3 has been the only transporter identified to inhibit root-to-shoot Cd translocation by vacuolar sequestration of $\mathrm{Cd}$ in roots (Ueno et al. 2010). OsHMA3 is also responsible for both $\mathrm{Zn}$ detoxification and storage by vacuolar sequestration of $\mathrm{Zn}$ (Cai et al. 2019). When overexpressed, OsHMA3 leads to more $\mathrm{Zn}$ sequestered into the vacuoles of root cells but does not affect the $\mathrm{Zn}$ accumulated in shoots and grains through up-regulating expression of OsZIP genes encoding transporters implicated in uptake, translocation and distribution of $\mathrm{Zn}$ (Sasaki et al. 2014; Ueno et al. 2010; Lu et al. 2019; Chen et al. 2021).

Here, overexpression of OsLCT2 enhances tolerance to excess $\mathrm{Cd}$ and reduces $\mathrm{Cd}$ accumulation in rice shoots and grains by limiting the amounts of $\mathrm{Cd}$ loaded into the xylem for root-to-shoot translocation. OsLCT2 was expressed in all tissues of the elongation and maturation zones of roots (Fig. 2d). These results raise a possibility that OsLCT2 overexpression restricts the radial movement of $\mathrm{Cd}$ into the xylem and subsequent translocation to the above-ground tissues by retaining a portion of $\mathrm{Cd}$ in the ER of root cells. We conducted heterologous expression of OsLCT2 in yeast. However, yeast could not grow when OsLCT2 was expressed in it, suggesting that expression of OsLCT2 has a lethal effect on yeast. The transport activity of OsLCT2 remains to be fully elucidated. 


\section{Overexpression of OsLCT2 May affect $\mathrm{Zn}$, Fe and Mn Accumulation in Rice}

Excessive expression of OsLCT2 also affected the rootto-shoot translocation of $\mathrm{Mn}, \mathrm{Fe}$ and $\mathrm{Zn}$, but not of $\mathrm{Cu}, \mathrm{Ca}$ and $\mathrm{Mg}$. This is supported by our evidence that the hydroponically-grown overexpression lines exhibited lower $\mathrm{Mn}$, Fe and $\mathrm{Zn}$ concentrations in shoots and xylem sap and lower rates of root-to-shoot translocation of these metals (Fig. 5f, k, l; Additional file 1: Fig. S3a-c), but similar $\mathrm{Cu}, \mathrm{Ca}$ and $\mathrm{Mg}$ concentrations in shoots and roots compared to those of WT plants (Fig. 5e-h). However, overexpressing OsLCT2 did not affect the accumulation of $\mathrm{Zn}, \mathrm{Fe}$ and $\mathrm{Mn}$ in straw (Fig. $4 \mathrm{~g}-\mathrm{i}$ ) and grains (Fig. $41-n$ ) at the maturity period of rice in field conditions. These results suggest that the effect of OsLCT2 overexpression on accumulation of $\mathrm{Mn}, \mathrm{Fe}$ and $\mathrm{Zn}$ is partially dependent on the environment or plant growth stage. We speculate the principal reason for this inconsistent performance of overexpression lines cultivated in nutrient solutions and field soils might be the differences of culture environments.

As essential micronutrients, $\mathrm{Mn}, \mathrm{Fe}$ and $\mathrm{Zn}$ are required for many biological processes, but excessive $\mathrm{Mn}, \mathrm{Fe}$ and $\mathrm{Zn}$ are toxic to plants (Bashir et al. 2019). Hence, it is likely that rice has evolutionarily developed efficient sensing systems and complex regulatory networks to maintain homeostasis of these metals. In the hydroponic condition, the concentrations of $\mathrm{Mn}, \mathrm{Fe}$ and $\mathrm{Zn}$ in the nutrient solution were easily controlled, and therefore supplies of these micronutrients were constant and sufficient for plant growth. Although OsLCT2 overexpression caused decreases in concentrations of $\mathrm{Mn}, \mathrm{Fe}$ and $\mathrm{Zn}$ in shoots (Fig. 5f), the amounts in these plants were sufficient for the normal growth and development (Fig. 5a). So it was likely that the signals of Mn, Fe, or Zn deficiency in the overexpression lines were too weak to induce adequate changes in expression of other metal transporters to offset the effect of OsLCT2 overexpression on reduction of metal concentrations in shoots. This was supported by our observation that the induction of OsZIP9 expression activated the uptake of $\mathrm{Zn}$, which increased the concentration of $\mathrm{Zn}$ in roots; however, the up-regulation of OsZIP9 expression was not enough to overcome the decrease of $\mathrm{Zn}$ concentration in shoots caused by OsLCT2 overexpression (Fig. 5f).

However, in field conditions, the contents of Mn, Fe and $\mathrm{Zn}$ in the soils fluctuated greatly and frequently, so metal ion homeostasis in plants overexpressing OsLCT2 was likely disturbed to a greater degree than in hydroponically cultivated plants overexpressing OsLCT2. Therefore, the transcription or protein levels of transporters implicated in uptake and translocation of $\mathrm{Mn}$, Fe and $\mathrm{Zn}$ may be regulated in field-grown plants to a greater extent to compensate for the lower $\mathrm{Mn}, \mathrm{Fe}$ and $\mathrm{Zn}$ concentrations in shoots resulting from OsLCT2 overexpression. Similarly, the overexpression of OsZIP9 also led to inconsistent $\mathrm{Zn}$ accumulation characteristics in rice seedlings growing under hydroponic conditions and in mature rice growing in field conditions (Yang et al. 2020).

We also performed hydroponic culture for OsLCT2 knockout lines and WT plants, treating them with $0.5 \mu \mathrm{M}$ Cd for two weeks and determined metal concentrations. The concentrations of $\mathrm{Cd}, \mathrm{Mn}, \mathrm{Zn}, \mathrm{Fe}$, and $\mathrm{Cu}$ in shoots and roots were similar between the knockout lines and WT plants (Additional file 2: Table S1). The lack of an obvious phenotype in the OsLCT2 knockout lines may be a result of the low expression level of OsLCT2 (Additional file 1: Fig. S5).

The localization of OsLCT2 to the ER when its coding gene was driven by $35 \mathrm{~S}$ promoter suggests that OsLCT2 overexpression might affect the distribution and homeostasis of $\mathrm{Zn}, \mathrm{Mn}$ and Fe in cells. Moreover, its overexpression was prone to reduce grain yield, which might be attributed to the higher sensitivity of reproductive cells to the disrupted homeostasis of intracellular metal. This also indicates that the promotion of OsLCT2 expression by a strong constitutive promoter is not an ideal strategy for breeding rice cultivars accumulating low levels of $\mathrm{Cd}$. Instead, enhancing expression of OSLCT2 by a root-specific promoter could be a better strategy for breeding rice cultivars with low $\mathrm{Cd}$ concentrations in rice grains.

\section{Conclusions}

A member of the low-affinity cation transporter $(L C T)$ family, OsLCT2 was cloned and characterized from rice. OsLCT2 was expressed in all tissues of the elongation and maturation zones in roots. Overexpression of OsLCT2 significantly reduced $\mathrm{Cd}$ concentrations in the shoots and grains by weakening xylem-mediated $\mathrm{Cd}$ translocation from roots to shoots in rice. Furthermore, its overexpression induced the expression of OsZIP9, a gene responsible for $\mathrm{Zn}$ uptake, and consequently increased $\mathrm{Zn}$ concentrations in roots. Our findings provide a new genetic resource to be manipulated to reduce $\mathrm{Cd}$ level in rice grains.

\section{Methods \\ Plant Material and Growth Conditions}

An indica cultivar Huazhan was used to determine OsLCT2 expression patterns and as the WT rice for phenotypic analyses. OsLCT2 overexpression lines, knockout lines and OSLCT2-promoter::GUS plants were generated in the background of cv. Huazhan.

Rice seedlings were grown hydroponically for OsLCT2 expression analysis and phenotypic analysis of overexpression plants. Rice seeds were soaked in tap water 
for $2 \mathrm{~d}$ and allowed $1 \mathrm{~d}$ for germination at $37^{\circ} \mathrm{C}$. Those seeds that germinated were sown into a floating plate with holes and placed on distilled water. After $7 \mathrm{~d}$, seedlings were transferred to plastic pots containing Yoshida nutrient solution as described previously (Yoshida et al. 1976). The $\mathrm{pH}$ of the nutrient solution was adjusted to 5.5 and renewed every $3 \mathrm{~d}$. Plants were grown in a growth chamber with a 14 -h light $\left(28^{\circ} \mathrm{C}\right) / 10$-h dark $\left(24{ }^{\circ} \mathrm{C}\right)$ photoperiod, $700 \mu \mathrm{mol} \mathrm{m}{ }^{-1} \mathrm{~s}^{-1}$ light intensity and $60-70 \%$ humidity.

OsLCT2-overexpressing lines, knockout lines and the WT were grown at a Cd-contaminated experimental paddy field in Hunan Province of China to evaluate their agronomic and grain ionomic traits. The soil $\mathrm{Cd}$ concentration was approximately $0.65 \mathrm{mg} / \mathrm{kg}$ and soil $\mathrm{pH}$ was 5.8. Rice seeds were sown on the seed bed and grown for 26 days, and then the seedlings were transplanted into the field, spaced at $17 \mathrm{~cm} \times 20 \mathrm{~cm}$. The field experiments were arranged in a randomized complete block design with three replications. The plants of each line were grown in 4 rows $\times 8$ plants in each plot. The fields were irrigated intermittently until the rice grains ripened.

\section{Cloning of OsLCT2 and Phylogenetic Analysis}

To obtain the full-length cDNA sequence of OsLCT2, total RNA was extracted from rice roots (cv. Huazhan) using the Plant RNA Kit (Omega), and the full-length cDNA sequence of OsLCT2 was achieved by RACE using the SMARTer ${ }^{\circledR}$ RACE $5^{\prime} / 3^{\prime}$ Kit (Clontech). The open reading frame of OsLCT2 was amplified by RT-PCR using specific primers (Additional file 2: Table S2) designed according to the sequence information obtained from the full-length OsLCT2 cDNA.

To perform a phylogenetic analysis, BLAST searches were performed to extract amino acid sequences of LCTlike proteins in different plant species from the NCBI (http://www.ncbi.nlm.nih.gov/) databases. LCT-like proteins in wild rice species were detected by BLAST searches of the UniProt database (https://www.uniprot. org/). The amino acid sequences were aligned using the ClustalW program, and the phylogenetic tree was constructed using MEGA7 (http://www.megasoftware.net/) by the neighbor-joining method with 1000 bootstrap trials. Trans-membrane domains were predicted with both the TMPRED program (https://embnet.vital-it.ch/softw are/TMPRED_form.html/) and the CCTOP program (http://cctop.enzim.ttk.mta.hu/). Genome sequence alignment was performed using MUMmer 4 (http:// mummer.sourceforge.net/). The haplotypes of OsLCT2 in the 3620 rice accessions were classified according to the SNPs within the CDS region using the MBKbase-rice database (http://www.mbkbase.org/rice/).

\section{Construction and Transformation of Plant Expression Vectors}

To generate the OsLCT2-promoter::GUS vector, the 2167-bp promoter sequence, upstream of the start codon, was amplified by PCR from the genomic DNA of rice cv. Huazhan and cloned into a Pst I/Nco I-digested pCAMBIA1301 vector using the CloneExpress ${ }^{\circledR}$ II One Step Cloning Kit (Vazyme). To generate the overexpression vector of OsLCT2, the ORF of OsLCT2 was amplified by PCR from the total cDNA of Huazhan and cloned into the Kpn I/Spe I-digested pTCK303 vector under the control of the ubiquitin promoter using the same recombination cloning method described above. The PCR primers are listed in Additional file 2: Table S2. A knockout construct of OsLCT2 was generated by CRISPR/Cas9 technology (Ma and Liu 2016), targeting two sequences at the third exon of OsLCT2 (Fig. 4b). All constructs were transformed into Huazhan using Agrobacterium-mediated transformation, which was conducted by Wuhan Biorun Biological Technology Co., Ltd. The homozygous overexpression lines were selected by hygromycin B screening and expression analysis. The homozygous knockout lines without Cas 9 were selected by PCR and DNA sequencing.

\section{Gene Expression Analysis}

Leaves, roots and the basal region $(2 \mathrm{~cm}$ above the roots) of rice seedlings (14 d old) grown in Yoshida nutrient solution were sampled for expression pattern analysis. The tissue of the seedlings with the highest OsLCT2 expression level was selected for use in the subsequent metal-response analyses. OsLCT2 expression in response to metal excess or deficiency was determined by exposing the one-week-old Huazhan seedlings to the Yoshida nutrient solutions without $\mathrm{Fe}, \mathrm{Mn}$, or $\mathrm{Zn}$, or with high concentrations of Fe (500 or $\left.1000 \mu \mathrm{M} \mathrm{FeSO}_{4}, \mathrm{pH} 4.5\right)$, $\mathrm{Mn}\left(100\right.$ or $\left.500 \mu \mathrm{M} \mathrm{MnCl}_{2}\right)$, or $\mathrm{Zn}\left(40\right.$ or $\left.100 \mu \mathrm{M} \mathrm{ZnSO}_{4}\right)$ for $7 \mathrm{~d}$, based on previous reports (Lee et al. 2007; Sasaki et al. 2011; Aung et al. 2018; Liu et al. 2019; Tan et al. 2020). The levels of metal deficiency in rice were determined using the appropriate marker genes (Zheng et al. 2012). To determine OsLCT2 expression in response to $\mathrm{Cd}$, two-week-old Huazhan seedlings were exposed to $0.5,2.5$ or $25 \mu \mathrm{M} \mathrm{CdCl}_{2}$ for $24 \mathrm{~h}$. Seedlings grown in standard Yoshida nutrient solution were used as controls. After $\mathrm{Cd}$ treatments, leaves, roots and basal regions were separated for RNA extraction. The OsLCT2 overexpression lines and WT plants were used to analyze the effect of OsLCT2 overexpression on the expression of OsZIP genes. Two-week-old rice seedlings grown hydroponically were exposed to 0 or $0.5 \mu \mathrm{M} \mathrm{CdCl}_{2}$ for $1 \mathrm{~d}$, and the roots were sampled for RNA extraction. 
The total RNA of samples was extracted and then converted to cDNA using the PrimeScript ${ }^{\mathrm{TM}}$ RT reagent kit with gDNA Eraser (Takara). qRT-PCR was conducted using TB Green ${ }^{\circledR}$ Premix Ex Taq ${ }^{\mathrm{TM}}$ II (TaKaRa) with a LightCycler 480II PCR instrument (Roche). The expression level of OsLCT2 and ten OsZIP genes were determined. Histone $H 3$ and Actin 1 were used as internal controls. All primers used for qRT-PCR are listed in Additional file 2: Table S2.

\section{GUS Histochemical Analysis}

A GUS histochemical analysis was performed on transgenic OsLCT2-promoter::GUS plants. The transgenic plant samples were soaked in fixation buffer for $45 \mathrm{~min}$ and then incubated in X-Gluc staining buffer at $37{ }^{\circ} \mathrm{C}$ overnight using GUS Stain Kit (Solarbio, G3060) following the manufacturer's instructions. The stained samples were held in $70 \%(\mathrm{v} / \mathrm{v})$ ethanol and observed under a stereomicroscope (Smart Zoom 5, Zeiss). To make paraffin sections, stained tissues were fixed in FAA (formalinacetic acid-50\% ethanol [1:1:18]), vacuum-infiltrated for $10 \mathrm{~min}$, then kept for $24 \mathrm{~h}$. The fixed samples were dehydrated in a graded ethanol series $(50 \%, 60 \%, 70 \%, 80 \%$, $90 \%, 95 \%, 100 \%)$ and cleared in a gradient xylene series (50\%, 75\%, 90\%, 100\%). The samples were then embedded in paraffin, sectioned to $8 \mu \mathrm{m}$ thickness using a microtome (HM340E, Thermo) and photographed under a microscope (Leica).

\section{Subcellular Localization Analysis}

A 16 amino-acid (aa) linker was synthesized and inserted into the pAN580 vector between Nhe I and BamH I restriction sites at the $\mathrm{N}$-terminus of eGFP in the pAN580 vector. The ORF sequence of OsLCT2 without the stop codon was amplified using total cDNA of Huazhan. The PCR product was then cloned into the Spe I/Nhe I-digested pAN580 vector with the linker to generate the 35S::OsLCT2-linker-eGFP vector. The 16 aa linker was also inserted into the pAN580 vector between $B g l$ II and Pst I restriction sites fused in-frame to the C-terminus of eGFP. The ORF sequence of OsLCT2 was cloned into the Pst I/EcoR I-digested pAN580 vector with the linker to generate the 35S::eGFP-linker-OsLCT2 vector. These vectors were constructed by recombination using the primers shown in Additional file 2: Table S2 with ClonExpress ${ }^{\circledR}$ II One Step Cloning Kit (Vazyme). The pAN580 vector containing the expression cassette of $35 S:: e G F P$ was used as a control. The 35S::OsLCT2linker-eGFP, 35S:: eGFP-linker-OsLCT2 or 35S:: eGFP construct was co-transformed with vector carrying 35S::HDEL-mCherry into rice protoplasts via polyethylene glycol-mediated transformation. All protoplasts were observed and imaged using a confocal laser scanning microscope (LSM 880, Zeiss) after incubation at $28{ }^{\circ} \mathrm{C}$ for 16 to $24 \mathrm{~h}$.

\section{Determination of Metal Concentrations in Plant Tissues}

Two-week-old seedlings of hydroponically-grown rice were exposed to a nutrient solution with either $0,0.5$ or $1 \mu \mathrm{M} \mathrm{CdCl}{ }_{2}$ for 14 days. Then the samples were washed with distilled water three times and separated into shoots and roots. Field-grown rice plants were harvested after grain ripening and separated into straw (shoot) and brown rice (grain).

All samples were dried at $70{ }^{\circ} \mathrm{C}$ for $3 \mathrm{~d}$, and then digested with an acid mixture of $\mathrm{HNO}_{3}: \mathrm{HClO}_{4}(6: 1[\mathrm{v} / \mathrm{v}])$ as described previously (Tang et al. 2017). The metal concentrations in the digest solutions were determined by inductively coupled plasma mass spectrometry (ICP-MS) (Agilent 7700 series, USA). The root-to-shoot translocation rates of metals were calculated as the amount of metal accumulated in the shoots as a percentage of the total amount of metal accumulated in the whole plant, as described by Miyadate et al. (2011).

\section{Collection and Analysis of xylem Sap}

After two weeks of Cd treatment, the 4-week-old seedlings of two OsLCT2-overexpressing and WT plants were cut with a razor at about $2 \mathrm{~cm}$ above the root-shoot junction. Xylem sap was collected from the cut surface for $1 \mathrm{~h}$ using micropipettes. The first drop of xylem sap emerging from the cut end was discarded to avoid contamination from the symplastic metal ions in the cells damaged by cutting. Xylem sap from 20 plants was combined as one biological replicate and three biological replicates were made. Metal concentrations in the xylem sap were determined by ICP-MS.

\section{Abbreviations \\ Cd: Cadmium; Mn: Manganese; Fe: Iron; Zn: Zinc; Ca: Calcium; K: Potas- sium; Mg: Magnesium; LCT: Low-affinity cation transporter; NRAMP: Natural resistance-associated macrophage protein; ZIP: Zinc-regulated/iron-regulated transporter-like protein; HMA: Heavy metal ATPase; ER: Endoplasmic reticulum; NCBI: National center for biotechnology information; RACE: Rapid amplifica- tion of CDNA ends; ORF: Open reading frame; GUS: $\beta$-Glucuronidase; HDEL: His-Asp-Glu-Leu; qRT-PCR: Quantitative real-time PCR; eGFP: Enhanced green fluorescent protein; SNPs: Single-nucleotide polymorphisms.}

\section{Supplementary Information}

The online version contains supplementary material available at https://doi. org/10.1186/s12284-021-00530-8.

Additional file 1. Supplemental Figures. Fig. S1. Genetic diversity of OSLCT2. Fig. S2. Agronomic traits of OSLCT2 overexpression lines and the WT grown in paddy fields. Fig. S3. Metal concentrations in shoots and roots of overexpression lines and the WT treated with Cd for 14 days. Fig. S4. Synteny analysis of regions encompassing OsLCT1 or OsLCT2 on 
chromosome 6 between O. sativa cv. Nipponbare and cv. Shuhui498. Fig. S5. qRT-PCR-based expression analysis of OsLCT2 in cv. Huazhan.

Additional file 2. Supplemental Tables. Table S1. Concentrations of metal in shoots and roots of OsLCT2 seedling ( $\mathrm{mg} / \mathrm{kg})$. Table S2. Primers used in the present study.

\section{Acknowledgements}

Not applicable.

\section{Authors' contributions}

L.T. and B.Z. designed the research. L.T., J.D., L.T., Z.J. Y.L., and Y.S. performed the experiments. CC provided technical support on subcellular localization. L.T., Q.L., B.M. and Y.H. analyzed the data. L.T. wrote the manuscript. C.C. and B.Z. revised the manuscript. All authors read and approved the final manuscript.

\section{Funding}

This work was supported by the Earmarked Fund for Modern Agro-Industry Technology System of China (Grant No. CARS-05-01), Changsha Municipal Natural Science Foundation (kq2007011), Research and Development Projects in Key Areas of Hunan Province (2020NK2043), and the Natural Science Foundation of Hunan Province (2018JJ3377).

\section{Availability of data and materials}

The datasets supporting the conclusions of this article are included within the article and its additional files.

\section{Declarations}

\section{Ethics approval and consent to participate}

Not applicable.

\section{Consent for Publication}

Not applicable.

\section{Competing interests}

The authors declare that they have no competing interest.

\section{Author details}

${ }^{1}$ State Key Laboratory of Hybrid Rice, Hunan Hybrid Rice Research Center, Changsha 410125, China. ${ }^{2}$ Longping Branch of Graduate School, Hunan University, Changsha 410125, China. ${ }^{3}$ Key Laboratory of Agro-Ecological Processes in Subtropical Region, Institute of Subtropical Agriculture, Chinese Academy of Sciences, Changsha 410125, China.

Received: 13 June 2021 Accepted: 12 October 2021

Published online: 24 October 2021

\section{References}

Ajeesh Krishna TP, Maharajan T, Victor Roch G, Ignacimuthu S, Antony Ceasar S (2020) Structure, function, regulation and phylogenetic relationship of ZIP family transporters of plants. Front Plant Sci 11:662

Aung MS, Masuda H, Kobayashi T, Nishizawa NK (2018) Physiological and transcriptomic analysis of responses to different levels of iron excess stress in various rice tissues. Soil Sci Plant Nutr 64:370-385

Bashir K, Seki M, Nishizawa NK (2019) The transport of essential micronutrients in rice. Mol Breed 39:168

Cai H, Huang S, Che J, Yamaji N, Ma JF (2019) The tonoplast-localized transporter OsHMA3 plays an important role in maintaining $\mathrm{Zn}$ homeostasis in rice. J Exp Bot 70:2717-2725

Chang JD, Huang S, Yamaji N, Zhang W, Ma JF, Zhao FJ (2020) OsNRAMP1 transporter contributes to cadmium and manganese uptake in rice. Plant Cell Environ 43:2476-2491

Chen HP, Wang P, Chang JD, Kopittke PM, Zhao FJ (2021) Producing Cd-safe rice grains in moderately and seriously $\mathrm{Cd}$-contaminated paddy soils. Chemosphere 267:128893
Chi Y, Li F, Tam NF, Liu C, Ouyang Y, Qi X, Li WC, Ye Z (2018) Variations in grain cadmium and arsenic concentrations and screening for stable low-accumulating rice cultivars from multi-environment trials. Sci Total Environ 643:1314-1324

Clemens S, Ma JF (2016) Toxic heavy metal and metalloid accumulation in crop plants and foods. Annu Rev Plant Biol 67:489-512

Clemens S, Antosiewicz DM, Ward JM, Schachtman DP, Schroeder JI (1998) The plant CDNA LCT1 mediates the uptake of calcium and cadmium in yeast. Proc Natl Acad Sci U S A 95:12043-12048

De Caroli M, Furini A, DalCorso G, Rojas M, Di Sansebastiano GP (2020) Endomembrane reorganization induced by heavy metals. Plants (basel) 9:482

Godt J, Scheidig F, Grosse-Siestrup C, Esche V, Groneberg DA (2006) The toxicity of cadmium and resulting hazards for human health. J Occup Med Toxicol 1:22

Hao X, Zeng M, Wang J et al (2018) A node-expressed transporter OsCCX2 is involved in grain cadmium accumulation of rice. Front Plant Sci 9:476

Hu Y, Cheng H, Tao S (2016) The challenges and solutions for cadmium-contaminated rice in China: a critical review. Environ Int 92-93:515-532

Huang S, Sasaki A, Yamaji N, Okada H, Mitani-Ueno N, Ma JF (2020) The ZIP transporter family member OsZIP9 contributes to root zinc uptake in rice under zinc-limited conditions. Plant Physiol 183:1224-1234

Ishikawa S, Ishimaru Y, Igura M et al (2012) Ion-beam irradiation, gene identification, and marker-assisted breeding in the development of low-cadmium rice. Proc Natl Acad Sci U S A 109:19166-19171

Kennedy G, Rinne K (1997) Molecular and functional characterization of a novel low-affinity cation transporter (LCT1) in higher plants. Proc Natl Acad Sci U S A 94:11079-11084

Lee S, An G (2009) Over-expression of Os/RT1 leads to increased iron and zinc accumulations in rice. Plant Cell Environ 32:408-416

Lee S, Kim YY, Lee Y, An G (2007) Rice P1B-type heavy-metal ATPase, OsHMA9, is a metal efflux protein. Plant Physiol 145:831-842

Liu XS, Feng SJ, Zhang BQ, Wang MQ, Cao HW, Rono JK, Chen X, Yang ZM (2019) OsZIP1 functions as a metal efflux transporter limiting excess zinc, copper and cadmium accumulation in rice. BMC Plant Biol 19:283

Lu C, Zhang L, Tang Z, Huang XY, Ma JF, Zhao FJ (2019) Producing cadmiumfree indica rice by overexpressing OsHMA3. Environ Int 126:619-626

Luo JS, Huang J, Zeng DL et al (2018) A defensin-like protein drives cadmium efflux and allocation in rice. Nat Commun 9:645

Ma X, Liu YG (2016) CRISPR/Cas9-based multiplex genome editing in monocot and dicot plants. Curr Protoc Mol Biol 115:31.36.31-31.36.21

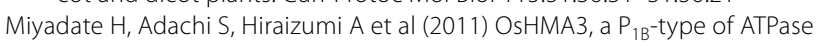
affects root-to-shoot cadmium translocation in rice by mediating efflux into vacuoles. New Phytol 189:190-199

Nakanishi H, Ogawa I, Ishimaru Y, Mori S, Nishizawa NK (2006) Iron deficiency enhances cadmium uptake and translocation mediated by the $\mathrm{Fe}^{2+}$ transporters OsIRT1 and OsIRT2 in rice. Soil Sci Plant Nutr 52:464-469

Nocito FF, Lancilli C, Dendena B, Lucchini G, Sacchi GA (2011) Cadmium retention in rice roots is influenced by cadmium availability, chelation and translocation. Plant Cell Environ 34:994-1008

Peng H, Wang K, Chen Z, Cao Y, Gao Q, Li Y, Li X, Lu H, Du H, Lu M, Yang X, Liang C (2020) MBKbase for rice: an integrated omics knowledgebase for molecular breeding in rice. Nucleic Acids Res 48:D1085-d1092

Sasaki A, Yamaji N, Xia J, Ma JF (2011) OsYSL6 is involved in the detoxification of excess manganese in rice. Plant Physiol 157:1832-1840

Sasaki A, Yamaji N, Yokosho K, Ma JF (2012) Nramp5 is a major transporter responsible for manganese and cadmium uptake in rice. Plant Cell 24:2155-2167

Sasaki A, Yamaji N, Ma JF (2014) Overexpression of OsHMA3 enhances Cd tolerance and expression of $\mathrm{Zn}$ transporter genes in rice. J Exp Bot 65:6013-6021

Satoh-Nagasawa N, Mori M, Nakazawa N, Kawamoto T, Nagato Y, Sakurai K, Takahashi H, Watanabe A, Akagi H (2012) Mutations in rice (Oryza sativa) heavy metal ATPase 2 (OsHMA2) restrict the translocation of zinc and cadmium. Plant Cell Physiol 53:213-224

Senoura T, Shimo H, Ishikawa S, Nishizawa NK (2011) The OsNRAMP1 iron transporter is involved in Cd accumulation in rice. J Exp Bot 62:4843-4850

Shao JF, Yamaji N, Shen RF, Ma JF (2017) The key to Mn homeostasis in plants: regulation of Mn transporters. Trends Plant Sci 22:215-224 
Sun C, Yang M, Li Y et al (2019) Comprehensive analysis of variation of cadmium accumulation in rice and detection of a new weak allele of OsHMA3. J Exp Bot 70:6389-6400

Takahashi R, Ishimaru Y, Shimo H, Ogo Y, Senoura T, Nishizawa NK, Nakanishi H (2012) The OsHMA2 transporter is involved in root-to-shoot translocation of $\mathrm{Zn}$ and Cd in rice. Plant Cell Environ 35:1948-1957

Tan L, Zhu Y, Fan T, Peng C, Wang J, Sun L, Chen C (2019) OsZIP7 functions in xylem loading in roots and inter-vascular transfer in nodes to deliver $\mathrm{Zn}$ / $\mathrm{Cd}$ to grain in rice. Biochem Biophys Res Commun 512:112-118

Tan L, Qu M, Zhu Y, Peng C, Wang J, Gao D, Chen C (2020) Zinc transporter5 and zinc transporter9 function synergistically in zinc/cadmium uptake. Plant Physiol 183:1235-1249

Tang L, Mao B, Li Y et al (2017) Knockout of OsNramp5 using the CRISPR/Cas9 system produces low $\mathrm{Cd}$-accumulating indica rice without compromising yield. Sci Rep 7:14438

Ueno D, Yamaji N, Kono I, Huang CF, Ando T, Yano M, Ma JF (2010) Gene limiting cadmium accumulation in rice. Proc Natl Acad Sci U S A 107:16500-16505

Uraguchi S, Fujiwara T (2013) Rice breaks ground for cadmium-free cereals. Curr Opin Plant Biol 16:328-334

Uraguchi S, Mori S, Kuramata M, Kawasaki A, Arao T, Ishikawa S (2009) Root-toshoot Cd translocation via the xylem is the major process determining shoot and grain cadmium accumulation in rice. J Exp Bot 60:2677-2688

Uraguchi S, Kamiya T, Sakamoto T et al (2011) Low-affinity cation transporter (OsLCT1) regulates cadmium transport into rice grains. Proc Natl Acad Sci U S A 108:20959-20964

Uraguchi S, Kamiya T, Clemens S, Fujiwara T (2014) Characterization of OsLCT1, a cadmium transporter from indica rice (Oryza sativa). Physiol Plant 151:339-347
Yamaji N, Xia J, Mitani-Ueno N, Yokosho K, Feng Ma J (2013) Preferential delivery of zinc to developing tissues in rice is mediated by P-type heavy metal ATPase OsHMA2. Plant Physiol 162:927-939

Yan J, Wang P, Wang P, Yang M, Lian X, Tang Z, Huang CF, Salt DE, Zhao FJ (2016) A loss-of-function allele of OsHMA3 associated with high cadmium accumulation in shoots and grain of japonica rice cultivars. Plant Cell Environ 39:1941-1954

Yan H, Xu W, Xie J et al (2019) Variation of a major facilitator superfamily gene contributes to differential cadmium accumulation between rice subspecies. Nat Commun 10:2562

Yang M, Zhang Y, Zhang L et al (2014) OsNRAMP5 contributes to manganese translocation and distribution in rice shoots. J Exp Bot 65:4849-4861

Yang M, Li Y, Liu Z et al (2020) A high activity zinc transporter OsZIP9 mediates zinc uptake in rice. Plant J 103:1695-1709

Yoshida S, Forno DA, Cock JH, Gomez KA (1976) Laboratory manual for physiological studies of rice: International Rice Research Institute.

Zhao F-J, Wang P (2019) Arsenic and cadmium accumulation in rice and mitigation strategies. Plant Soil 446:1-21

Zheng L, Yamaji N, Yokosho K, Ma JF (2012) YSL16 is a phloem-localized transporter of the copper-nicotianamine complex that is responsible for copper distribution in rice. Plant Cell 24:3767-3782

\section{Publisher's Note}

Springer Nature remains neutral with regard to jurisdictional claims in published maps and institutional affiliations.

\section{Submit your manuscript to a SpringerOpen ${ }^{\circ}$ journal and benefit from:}

- Convenient online submission

- Rigorous peer review

- Open access: articles freely available online

- High visibility within the field

- Retaining the copyright to your article

Submit your next manuscript at $\boldsymbol{\Delta}$ springeropen.com 